\title{
Dynamic robust duality in utility maximization
}

\author{
Bernt $\varnothing k s e n d a l^{1,2} \quad$ Agnès Sulem ${ }^{3,4}$
}

24 August 2015

\begin{abstract}
A celebrated financial application of convex duality theory gives an explicit relation between the following two quantities:

(i) The optimal terminal wealth $X^{*}(T):=X_{\varphi^{*}}(T)$ of the problem to maximize the expected $U$-utility of the terminal wealth $X_{\varphi}(T)$ generated by admissible portfolios $\varphi(t) ; 0 \leq t \leq T$ in a market with the risky asset price process modeled as a semimartingale;

(ii) The optimal scenario $\frac{d Q^{*}}{d P}$ of the dual problem to minimize the expected $V$-value of $\frac{d Q}{d P}$ over a family of equivalent local martingale measures $Q$, where $V$ is the convex conjugate function of the concave function $U$.

In this paper we consider markets modeled by Itô-Lévy processes. In the first part we use the maximum principle in stochastic control theory to extend the above relation to a dynamic relation, valid for all $t \in[0, T]$. We prove in particular that the optimal adjoint process for the primal problem coincides with the optimal density process, and that the optimal adjoint process for the dual problem coincides with the optimal wealth process; $0 \leq t \leq T$. In the terminal time case $t=T$ we recover the classical duality connection above.

We get moreover an explicit relation between the optimal portfolio $\varphi^{*}$ and the optimal measure $Q^{*}$. We also obtain that the existence of an optimal scenario is equivalent to the replicability of a related $T$-claim.

In the second part we present robust (model uncertainty) versions of the optimization problems in (i) and (ii), and we prove a similar dynamic relation between them. In particular, we show how to get from the solution of one of the problems to the other. We illustrate the results with explicit examples.
\end{abstract}

\footnotetext{
${ }^{1}$ Department of Mathematics, University of Oslo, P.O. Box 1053 Blindern, N-0316 Oslo, Norway, email: oksendal@math.uio.no. The research leading to these results has received funding from the European Research Council under the European Community's Seventh Framework Programme (FP7/2007-2013) / ERC grant agreement no [228087].

${ }^{2}$ Norwegian School of Economics, Helleveien 30, N-5045 Bergen, Norway.

${ }^{3}$ INRIA Paris-Rocquencourt, Domaine de Voluceau, Rocquencourt, BP 105, Le Chesnay Cedex, 78153, France, email: agnes.sulem@inria.fr

${ }^{4}$ Université Paris-Est, F-77455 Marne-la-Vallée, France.
} 
Keywords: Utility maximization, Itô-Lévy market, duality method, stochastic control, maximum principles, backward stochastic differential equations, replicability, optimal scenario, optimal portfolio, robust duality, robust portfolio optimization.

MSC(2012): Primary 60H10, 93E20. Secondary 91B70, 46N10.

\section{Introduction}

The purpose of this paper is to use stochastic control theory to obtain new results on the connections between the primal, utility maximization portfolio problem and its convex dual, both in the non-robust and the robust (worst case scenario/multiple-priors) setting. This approach allows us to get more detailed information about the connection between the primal and the dual problem. In particular, we show that the optimal wealth process of the primal problem coincides with the optimal adjoint process for the dual problem. This generalizes results that have been obtained earlier by using convex duality theory.

First, let us briefly recall the main results from the duality method in utility maximization, as presented in e.g. [8]: Let $U:[0, \infty] \rightarrow \mathbb{R}$ be a given utility function, assumed to be strictly increasing, strictly concave, continuously differentiable $\left(C^{1}\right)$ and satisfying the Inada conditions:

$$
\begin{aligned}
U^{\prime}(0) & =\lim _{x \rightarrow 0^{+}} U^{\prime}(x)=\infty \\
U^{\prime}(\infty) & =\lim _{x \rightarrow \infty} U^{\prime}(x)=0 .
\end{aligned}
$$

Let $S(t)=S(t, \omega) ; 0 \leq t \leq T, \omega \in \Omega$, represent the discounted unit price of a risky asset at time $t$ in a financial market. We assume that $S(t)$ is a semimartingale on a filtered probability space $\left(\Omega, \mathcal{F}, \mathbb{F}:=\left\{\mathcal{F}_{t}\right\}_{0 \leq t \leq T}, P\right)$. Let $\varphi(t)$ be an $\mathbb{F}$-predictable portfolio process, giving the number of units held of the risky asset at time $t$. If $\varphi(t)$ is self-financing, the corresponding wealth process $X(t):=X_{\varphi}^{x}(t)$ is given by

$$
X(t)=x+\int_{0}^{t} \varphi(s) d S(s) ; 0 \leq t \leq T,
$$

where $T \geq 0$ is a fixed terminal time and $x>0$ is the initial value of the wealth. We say that $\varphi$ is admissible and write $\varphi \in \mathcal{A}$ if the integral in (1.1) converges and

$$
X_{\varphi}^{x}(t)>0 \text { for all } t \in[0, T] \text {, a.s.. }
$$

The classical optimal portfolio problem is to find $\varphi^{*} \in \mathcal{A}$ (called an optimal portfolio) such that

$$
u(x):=\sup _{\varphi \in \mathcal{A}} E\left[U\left(X_{\varphi}^{x}(T)\right)\right]=E\left[U\left(X_{\varphi^{*}}^{x}(T)\right)\right] .
$$

The duality approach to this problem is as follows: Let

$$
V(y):=\sup _{x>0}\{U(x)-x y\} ; y>0
$$


be the convex conjugate function of $U$. Then it is well-known that $V$ is strictly convex, decreasing, $C^{1}$ and satisfies

$$
V^{\prime}(0)=-\infty, V^{\prime}(\infty)=0, V(0)=U(\infty) \text { and } V(\infty)=U(0)
$$

Moreover,

$$
U(x)=\inf _{y>0}\{V(y)+x y\} ; x>0,
$$

and

$$
U^{\prime}(x)=y \Leftrightarrow x=-V^{\prime}(y) .
$$

Let $\mathcal{M}$ be the set of probability measures $Q$ which are equivalent local martingale measures (ELMM), in the sense that $Q$ is equivalent to $P$ and $S(t)$ is a local martingale with respect to $Q$. We assume that $\mathcal{M} \neq \emptyset$, which means absence of arbitrage opportunities on the financial market. The dual problem to (1.3) is for given $y>0$ to find $Q^{*} \in \mathcal{M}$ (called an optimal scenario measure) such that

$$
v(y):=\inf _{Q \in \mathcal{M}} E\left[V\left(y \frac{d Q}{d P}\right)\right]=E\left[V\left(y \frac{d Q^{*}}{d P}\right)\right] .
$$

One of the main results in [8] is that, under some conditions, $\varphi^{*}$ and $Q^{*}$ both exist and they are related by

$$
U^{\prime}\left(X_{\varphi^{*}}^{x}(T)\right)=y \frac{d Q^{*}}{d P} \quad \text { with } y=u^{\prime}(x)
$$

i.e.

$$
X_{\varphi^{*}}^{x}(T)=-V^{\prime}\left(y \frac{d Q^{*}}{d P}\right) \quad \text { with } x=-v^{\prime}(y) .
$$

In this paper we extend this result to a dynamic identity between processes by using stochastic control theory. We work in the slightly more special market setting with a risky asset price $S(t)$ described by an Itô-Lévy process. This enables us to use the machinery of the maximum principle and backward stochastic differential equations (BSDE) driven by Brownian motion $B(t)$ and a compensated Poisson random measure $\tilde{N}(d t, d \zeta) ; t \geq 0 ; \zeta \in$ $\mathbb{R}_{0}:=\mathbb{R} \backslash\{0\}$. (We refer to e.g. [13] for more information about the maximum principle). Our approach has the advantage that it gives a dynamic relation between the optimal scenario in the dual formulation and the optimal portfolio in the primal formulation:

In particular, in Section 3 we prove that

$$
\hat{X}(t)=\hat{p}_{2}(t) ; t \in[0, T]
$$

where $\hat{X}(t)$ is the optimal wealth process and $\hat{p}_{2}(t)$ is the adjoint process for the dual problem. When $t=T$ this gives the classical duality result above, namely

$$
\hat{X}(T)=-V^{\prime}\left(y \frac{d Q^{*}}{d P}\right)\left(=\hat{p}_{2}(T)\right)
$$


A similar result is obtained for the optimal density process for the dual problem. As a step on the way, we prove in Section 2.1 a result of independent interest, namely that the existence of an optimal scenario is equivalent to the replicability of a related $T$-claim.

Then in Section 4 we extend the discussion to robust (model uncertainty) optimal portfolio problems. More precisely, we formulate robust versions of the primal problem (1.3) and of the dual problem (1.8), we establish a corresponding dynamic identity between processes and we show explicitly how to get from the solution of one to the solution of the other.

This paper addresses duality of robust utility maximization problems entirely by means of stochastic control methods, but there are several papers of related interest based on convex duality methods, see e.g. the survey paper [4] and the references therein. We also refer the reader to [16] where the author uses convex duality to study utility maximization under model uncertainty (multiple prior) and obtains a BSDE characterization of the optimal wealth process in markets driven by Brownian motion. In [6], a robust dual characterization of the robust primal utility maximization problem is obtained by convex duality methods. The dual formulation obtained is similar to ours, but there is no BSDE connection.

None of the above papers deal with a dynamic duality.

\section{Dynamic duality in utility maximization}

\subsection{Optimal portfolio, optimal scenario and replicability}

We now specialize the setting described in Section 1 as follows: Suppose the financial market has a risk free asset with unit price $S_{0}(t)=1$ for all $t$ and a risky asset with price $S(t)$ given by

$$
\left\{\begin{array}{l}
d S(t)=S\left(t^{-}\right)\left(b(t) d t+\sigma(t) d B(t)+\int_{\mathbb{R}} \gamma(t, \zeta) \tilde{N}(d t, d \zeta)\right) ; 0 \leq t \leq T \\
S(0)>0
\end{array}\right.
$$

where $b(t), \sigma(t)$ and $\gamma(t, \zeta)$ are predictable processes satisfying $\gamma>-1$ and

$$
E\left[\int_{0}^{T}\left\{|b(t)|+\sigma^{2}(t)+\int_{\mathbb{R}} \gamma^{2}(t, \zeta) \nu(d \zeta)\right\} d t\right]<\infty .
$$

Here $B(t)$ and $\tilde{N}(d t, d \zeta):=N(d t, d \zeta)-\nu(d \zeta) d t$ is a Brownian motion and an independent compensated Poisson random measure, respectively, on a filtered probability space $\left(\Omega, \mathcal{F}, \mathbb{F}:=\left\{\mathcal{F}_{t}\right\}_{0<t<T}, P\right)$ satisfying the usual conditions, $P$ is a reference probability measure and $\nu$ is the Lévy measure of $N$.

Let $\varphi(t)$ be a self financing portfolio and let $X(t):=X_{\varphi}^{x}(t)$ be the corresponding wealth process given by

$$
\left\{\begin{array}{l}
d X(t)=\varphi(t) S\left(t^{-}\right)\left[b(t) d t+\sigma(t) d B(t)+\int_{\mathbb{R}} \gamma(t, \zeta) \tilde{N}(d t, d \zeta)\right] ; 0 \leq t \leq T \\
X(0)=x>0 .
\end{array}\right.
$$


Definition 2.1 (Admissible Portfolios) Let $\varphi$ be an $\mathbb{F}$-predictable, S-integrable process. We say that $\varphi$ is admissible if

$$
\begin{aligned}
& X(t)>0 \text { for all } t \in[0, T], \text { a.s. } \\
& E\left[\int_{0}^{T} \varphi(t)^{2} S(t)^{2}\left\{b(t)^{2}+\sigma^{2}(t)+\int_{\mathbb{R}} \gamma^{2}(t, \zeta) \nu(d \zeta)\right\} d t\right]<\infty, \\
& E\left[\int_{0}^{T}|X(t)|^{2} d t\right]<\infty \\
& E\left[U^{\prime}(X(T))^{2}\right]<\infty
\end{aligned}
$$

We denote by $\mathcal{A}$ the set of admissible portfolios. Conditions (2.4), (2.5) are needed for the application of the maximum principles. See Appendix A.

As in (1.3), for given $x>0$, we want to find $\varphi^{*} \in \mathcal{A}$ such that

$$
u(x):=\sup _{\varphi \in \mathcal{A}} E\left[U\left(X_{\varphi}^{x}(T)\right)\right]=E\left[U\left(X_{\varphi^{*}}^{x}(T)\right)\right] .
$$

We consider the family $\mathcal{M}$ of equivalent local martingale measures (ELMM) that can be represented by means of the family of positive measures $Q=Q_{\theta}$ of the form

$$
d Q_{\theta}(\omega)=G_{\theta}(T) d P(\omega) \text { on } \mathcal{F}_{T},
$$

where

$$
\left\{\begin{array}{l}
d G_{\theta}(t)=G_{\theta}\left(t^{-}\right)\left[\theta_{0}(t) d B(t)+\int_{\mathbb{R}} \theta_{1}(t, \zeta) \tilde{N}(d t, d \zeta)\right] ; 0 \leq t \leq T \\
G_{\theta}(0)=y>0
\end{array}\right.
$$

and $\theta=\left(\theta_{0}, \theta_{1}\right)$ is a predictable process satisfying the conditions

$$
E\left[\int_{0}^{T}\left\{\theta_{0}^{2}(t)+\int_{\mathbb{R}} \theta_{1}^{2}(t, \zeta) \nu(d \zeta)\right\} d t\right]<\infty, \theta_{1}(t, \zeta)>-1 \quad \text { a.s. }
$$

and

$$
b(t)+\sigma(t) \theta_{0}(t)+\int_{\mathbb{R}} \gamma(t, \zeta) \theta_{1}(t, \zeta) \nu(d \zeta)=0 ; t \in[0, T]
$$

If $y=1$ this condition implies that $Q_{\theta}$ is an ELMM for this market. See e.g. 13, Chapter $1]$.

Remark 2.2 The set $\mathcal{M}$ with $y=1$ is contained in the set ELMM. Note, however, that there are ELMM's which are not of the above form. But $\mathcal{M}$ is the family we choose to work with, and all our results are proved for this family of measures.

We let $\Theta$ denote the set of all $\mathbb{F}$-predictable processes $\theta=\left(\theta_{0}, \theta_{1}\right)$ satisfying (2.9) $-(2.10)$. The dual problem corresponding to (1.8) is for given $y>0$ to find $\hat{\theta} \in \Theta$ and $v(y)$ such that

$$
-v(y):=\sup _{\theta \in \Theta} E\left[-V\left(G_{\theta}^{y}(T)\right)\right]=E\left[-V\left(G_{\hat{\theta}}^{y}(T)\right)\right] .
$$


We will use two stochastic maximum principles for stochastic control to study the problem (2.11) and relate it to (2.6). We refer to Appendix A for a presentation of these principles and to [17] for more information about backward stochastic differential equations (BSDEs) with jumps.

We recall the existence and uniqueness result for BSDEs with jumps, due to Tang and Li (1994) (see [20]). If $T>0, F \in L^{2}\left(\mathcal{F}_{T}\right)$, and $g$ is a Lipschitz driver, then there exists a unique solution $\in S^{2} \times H^{2} \times H_{\nu}$ of the BSDE with jumps

$$
\begin{aligned}
& d p(t)=-g(t, p(t), q(t), r(t, \cdot)) d t+q(t) d B(t)+\int_{\mathbb{R}} r(t, \zeta) \tilde{N}(d t, d \zeta) ; 0 \leq t \leq T \\
& p(T)=F
\end{aligned}
$$

where

- $S^{2}$ is the set of real-valued càdlàg adapted processes $\phi$ with $E\left(\sup _{0 \leq t \leq T}\left|\phi_{t}\right|^{2}\right)<\infty$.

- $H^{2}$ is the set of real-valued predictable processes $\phi$ such that $E\left[\left(\int_{0}^{T} \phi_{t}^{2} d t\right)\right]<\infty$,

- $H_{\nu}^{2}$ is the set of predictable processes $\ell$ such that $E\left[\left(\int_{0}^{T}\left(\int_{\mathbb{R}}|\ell(t, \zeta)|^{2} \nu(d \zeta)\right) d t\right)\right]<\infty$.

From now on, when we say that a process triple $(p(t), q(t), r(t, \zeta))$ satisfies a BSDE of the form (2.12), it is tacitly understood that $(p, q, r) \in S^{2} \times H^{2} \times H_{\nu}$.

We first prove two auxiliary results, the first of which may be regarded as a special case of Proposition 4.4 in [5].

Proposition 2.3 (Primal problem and associated constrained FBSDE) Let $\hat{\varphi}$ in $\mathcal{A}$. Then $\hat{\varphi}$ is optimal for the primal problem (2.6) if and only if the (unique) solution $X$, $\left(\hat{p}_{1}, \hat{q}_{1}, \hat{r}_{1}\right)$ in $S^{2} \times H^{2} \times H_{\nu}^{2}$ of the FBSDE consisting of the SDE (2.3) and the BSDE

$$
\left\{\begin{array}{l}
d \hat{p}_{1}(t)=\hat{q}_{1}(t) d B(t)+\int_{\mathbb{R}} \hat{r}_{1}(t, \zeta) \tilde{N}(d t, d \zeta) ; 0 \leq t \leq T \\
\hat{p}_{1}(T)=U^{\prime}\left(X_{\hat{\varphi}}^{x}(T)\right)
\end{array}\right.
$$

$\{$ equa2.13\}

satisfies the equation

$$
b(t) \hat{p}_{1}(t)+\sigma(t) \hat{q}_{1}(t)+\int_{\mathbb{R}} \gamma(t, \zeta) \hat{r}_{1}(t, \zeta) \nu(d \zeta)=0 ; t \in[0, T] .
$$

Proof. (i) The Hamiltonian corresponding to the primal problem is given by

$$
H_{1}(t, x, \varphi, p, q, r)=\varphi S\left(t^{-}\right)\left(b(t) p+\sigma(t) q+\int_{\mathbb{R}} \gamma(t, \zeta) r(\zeta) \nu(d \zeta)\right) .
$$

Assume $\hat{\varphi} \in \mathcal{A}$ is optimal for the primal problem (2.6). Then by the necessary maximum principle (Theorem A.2), we have

$$
\left.\frac{\partial H_{1}}{\partial \varphi}\left(t, x, \varphi, \hat{p}_{1}(t), \hat{q}_{1}(t), \hat{r}_{1}(t, \cdot)\right)\right|_{\varphi=\hat{\varphi}(t)}=0,
$$


where $\left(\hat{p}_{1}, \hat{q}_{1}, \hat{r}_{1}\right)$ satisfies (2.13) , since $\frac{\partial H_{1}}{\partial x}\left(t, x, \varphi, \hat{p}_{1}(t), \hat{q}_{1}(t), \hat{r}_{1}(t, \cdot)\right)=0$. This implies (2.14).

(ii) Conversely, suppose the solution $\left(\hat{p}_{1}, \hat{q}_{1}, \hat{r}_{1}\right)$ of the BSDE (2.13) satisfies (2.14). Then $\hat{\varphi}$, with the associated $\left(\hat{p}_{1}, \hat{q}_{1}, \hat{r}_{1}\right)$ satisfies the conditions for the sufficient maximum principle (Theorem A.1) with the additional feature of a constraint. See (2.21) below. We conclude that $\hat{\varphi}$ is optimal.

Remark 2.4 The BSDE (2.13) is linear, and hence it is well known that it has a unique solution $(p, q, r)$ for every choice of $X_{\varphi}^{x}(T)$. See e.g. [17], [18]. We are seeking $\hat{\varphi}$ such that the corresponding solution $(\hat{p}, \hat{q}, \hat{r})$ of (2.13) also satisfies (2.14).

Remark 2.5 By (2.13) we have $\hat{p}_{1}(t)=E\left[U^{\prime}\left(X_{\hat{\varphi}}^{x}(T)\right) \mid \mathcal{F}_{t}\right]>0$ for all $t$ in $[0, T]$, and if we divide equation (2.14) throughout by $\hat{p}_{1}(t)$ we get

$$
b(t)+\sigma(t) \hat{\theta}_{0}(t)+\int_{\mathbb{R}} \gamma(t, \zeta) \hat{\theta}_{1}(t, \zeta) \nu(d \zeta)=0 ; t \in[0, T]
$$

$\{$ eq2.13c $\}$

where

$$
\hat{\theta}_{0}(t):=\frac{\hat{q}_{1}(t)}{\hat{p}_{1}(t)} ; \quad \hat{\theta}_{1}(t, \zeta):=\frac{\hat{r}_{1}(t, \zeta)}{\hat{p}_{1}(t)}, t \in[0, T] .
$$

By the Girsanov theorem this is saying that if we define the measure $Q_{\left(\hat{\theta}_{0}, \hat{\theta}_{1}\right)}$ as in (2.7),(2.8) with $y=1$, then $Q_{\left(\hat{\theta}_{0}, \hat{\theta}_{1}\right)}$ is an ELMM for the market described by (2.1).

We now turn to the dual problem (2.11):

Proposition 2.6 (Dual problem and associated constrained FBSDE) Let $\hat{\theta} \in \Theta$. Then $\hat{\theta}$ is an optimal scenario for the dual problem (2.11) if and only if the solution $G_{\hat{\theta}},\left(\hat{p}_{2}, \hat{q}_{2}, \hat{r}_{2}\right)$ in $S^{2} \times H^{2} \times H_{\nu}^{2}$ of the FBSDE consisting of the FSDE (2.8) and BSDE

$$
\left\{\begin{array}{l}
d \hat{p}_{2}(t)=K\left(\hat{q}_{2}, \hat{r}_{2}\right)(t)\left[b(t) d t+\sigma(t) d B(t)+\int_{\mathbb{R}} \gamma(t, \zeta) \tilde{N}(d t, d \zeta)\right] \\
\hat{p}_{2}(T)=-V^{\prime}\left(G_{\theta}^{y}(T)\right)
\end{array}\right.
$$

where

$$
K(q, r)(t):=\frac{q(t)}{\sigma(t)} \chi_{\sigma(t) \neq 0}+\frac{r(t, \zeta)}{\gamma(t, \zeta)} \chi_{\sigma(t)=0, \gamma(t, \zeta) \neq 0}
$$

also satisfies

$$
-\hat{q}_{2}(t) \gamma(t, \zeta)+\sigma(t) \hat{r}_{2}(t, \zeta)=0 ; 0 \leq t \leq T .
$$

Proof. We may regard the problem (2.11) as a stochastic control problem in the control process $\theta$ with the constraint (2.10). To solve this problem we use the well-known Lagrange multiplier technique. Thus we define the Hamiltonian $H_{2}^{L}$ by

$$
H_{2}^{L}\left(\theta_{0}, \theta_{1}, L\right):=g \theta_{0} q+g \int_{\mathbb{R}} \theta_{1}(\zeta) r(\zeta) \nu(d \zeta)+L(t)\left(b(t)+\sigma(t) \theta_{0}+\int_{\mathbb{R}} \gamma(t, \zeta) \theta_{1}(\zeta) \nu(d \zeta)\right)
$$


where $L(t)$ is the Lagrange multiplier process. Maximizing $H_{2}^{L}$ over all $\theta_{0}$ and $\theta_{1}$ gives the following first order conditions

$$
g q+L(t) \sigma(t)=0 ; \quad g r(\cdot)+L(t) \gamma(t, \cdot)=0 .
$$

Since $g=G_{\theta}(t) \neq 0$, we can write these as follows:

$$
q(t)=-\frac{L(t)}{G_{\theta}(t)} \sigma(t) ; r(t, \zeta)=-\frac{L(t)}{G_{\theta}(t)} \gamma(t, \zeta) .
$$

The adjoint equation becomes:

$$
\left\{\begin{aligned}
d p(t) & =-\frac{L(t)}{G_{\theta}(t)}\left[\left\{-\theta_{0}(t) \sigma(t)-\int_{\mathbb{R}} \theta_{1}(t, \zeta) \gamma(t, \zeta) \nu(d \zeta)\right\} d t\right. \\
& \left.+\sigma(t) d B(t)+\int_{\mathbb{R}} \gamma(t, \zeta) \tilde{N}(d t, d \zeta)\right] ; 0 \leq t \leq T \\
p(T) & =-V^{\prime}\left(G_{\theta}(T)\right) .
\end{aligned}\right.
$$

In view of (2.10) this can be written

$$
\left\{\begin{array}{l}
d p(t)=-\frac{L(t)}{G_{\theta}(t)}\left[b(t) d t+\sigma(t) d B(t)+\int_{\mathbb{R}} \gamma(t, \zeta) \tilde{N}(d t, d \zeta)\right] ; 0 \leq t \leq T \\
p(T)=-V^{\prime}\left(G_{\theta}(T)\right)
\end{array}\right.
$$

Note that

$$
\begin{gathered}
\text { If } \sigma(t) \neq 0 \text { then }-\frac{L(t)}{G_{\theta}(t)}=\frac{q(t)}{\sigma(t)} \\
\text { If } \gamma(t, \zeta) \neq 0 \text { then }-\frac{L(t)}{G_{\theta}(t)}=\frac{r(t, \zeta)}{\gamma(t, \zeta)}
\end{gathered}
$$

If $\sigma(t)=\gamma(t, \zeta)=0$, then by (2.22) we have $q(t)=r(t, \zeta)=0$ and hence we have $d p(t)=0$. Therefore, with $K(q, r)(t)$ defined as in (2.19), we get by (2.24)

$$
\left\{\begin{array}{l}
d p(t)=K(q, r)(t)\left[b(t) d t+\sigma(t) d B(t)+\int_{\mathbb{R}} \gamma(t, \zeta) \tilde{N}(d t, d \zeta)\right] ; 0 \leq t \leq T \\
p(T)=-V^{\prime}\left(G_{\theta}(T)\right)
\end{array}\right.
$$

By combining the two equations of (2.22) we get (2.20). This completes the proof of the necessary part.

The sufficient part follows from the fact that the functions $g \rightarrow-V(g)$ and

$$
g \rightarrow \sup _{\theta_{0}, \theta_{1}} H_{2}^{L}\left(t, g, \theta_{0}, \theta_{1}, \hat{p}_{2}(t), \hat{q}_{2}(t), \hat{r}_{2}(t, \cdot)\right)
$$

are concave.

We deduce as a by-product the following results of independent interest which relates the existence of a solution of the dual problem to the replication of a related $T$-claim. 
Proposition 2.7 For given $y>0$ and $\hat{\theta} \in \Theta$ the following are equivalent:

(i)

$$
\sup _{\theta \in \Theta} E\left[-V\left(G_{\theta}^{y}(T)\right)\right]=E\left[-V\left(G_{\hat{\theta}}^{y}(T)\right)\right]<\infty .
$$

(ii) The claim $F:=-V^{\prime}\left(G_{\hat{\theta}}^{y}(T)\right)$ is replicable, with initial value $x=\hat{p}_{2}(0)$, where $\left(\hat{p}_{2}, \hat{q}_{2}, \hat{r}_{2}\right)$ solves

$$
\left\{\begin{array}{l}
d \hat{p}_{2}(t)=K\left(\hat{q}_{2}, \hat{r}_{2}\right)(t)\left[b(t) d t+\sigma(t) d B(t)+\int_{\mathbb{R}} \gamma(t, \zeta) \tilde{N}(d t, d \zeta)\right] ; 0 \leq t \leq T \\
\hat{p}_{2}(T)=-V^{\prime}\left(G_{\hat{\theta}}^{y}(T)\right)
\end{array}\right.
$$

Moreover, if (i) or (ii) holds, then

$$
\hat{\varphi}(t):=\frac{K\left(\hat{q}_{2}, \hat{r}_{2}\right)(t)}{S\left(t^{-}\right)}
$$

is a replicating portfolio for $F:=-V^{\prime}\left(G_{\hat{\theta}}^{y}(T)\right)$, where $\left(\hat{p}_{2}, \hat{q}_{2}, \hat{r}_{2}\right)$ is the solution of the BSDE (2.28).

Proof. $\quad(\mathrm{i}) \Rightarrow$ (ii): We have already proved that (i) implies (2.28). This equation states that the contingent claim $F:=-V^{\prime}\left(G_{\hat{\theta}}^{y}(T)\right)$ is replicable, with replicating portfolio $\hat{\varphi}(t)$ given by (2.29) and initial value $x=\hat{p}_{2}(0)$. Note that $\hat{p}_{2}(t)>0$ for all $t$, since $V$ is strictly decreasing so $-V^{\prime}\left(G_{\hat{\theta}}^{y}(T)\right)>0$.

(ii) $\Rightarrow$ (i): Suppose $F:=-V^{\prime}\left(G_{\hat{\theta}}^{y}(T)\right)$ is replicable with initial value $x=\hat{p}_{2}(0)$, and let $\varphi \in \mathcal{A}$ be a replicating portfolio. Then $X(t)=X_{\varphi}^{x}(t)$ satisfies the equation

$$
\left\{\begin{array}{l}
d X(t)=\varphi(t) S\left(t^{-}\right)\left[b(t) d t+\sigma(t) d B(t)+\int_{\mathbb{R}} \gamma(t, \zeta) \tilde{N}(d t, d \zeta)\right] ; 0 \leq t \leq T \\
X(T)=-V^{\prime}\left(G_{\theta}^{y}(T)\right) .
\end{array}\right.
$$

Define

$$
\hat{p}(t):=X(t), \hat{q}(t):=\varphi(t) \sigma(t) S\left(t^{-}\right) \text {and } \hat{r}(t, \zeta):=\varphi(t) \gamma(t, \zeta) S\left(t^{-}\right) .
$$

They satisfy the relation (2.20). Moreover, by (2.31) we get

$$
\varphi(t) S\left(t^{-}\right)=K(q, r)(t)
$$

$\{$ eq2. $21 \mathrm{a}\}$

Therefore, from (2.30) we get that $(\hat{p}, \hat{q}, \hat{r})$ satisfies the BSDE

$$
\left\{\begin{array}{l}
d \hat{p}(t)=K(\hat{q}, \hat{r})(t)\left[b(t) d t+\hat{q}(t) d B(t)+\int_{\mathbb{R}} \hat{r}(t, \zeta) \tilde{N}(d t, d \zeta)\right] ; 0 \leq t \leq T \\
\hat{p}(T)=-V^{\prime}\left(G_{\hat{\theta}}^{y}(T)\right) .
\end{array}\right.
$$

We conclude that $\hat{p}(t)=X(t)=\hat{p}_{2}(t)$. Hence (i) holds, by Proposition 2.6.

The last statement follows from (2.32). 


\subsection{Relations between optimal scenario and optimal portfolio}

We proceed to show that the method above actually gives a connection between an optimal scenario $\hat{\theta} \in \Theta$ for the dual problem (2.11) and an optimal portfolio $\hat{\varphi} \in \mathcal{A}$ for the primal problem (2.6).

Theorem 2.8 a) Suppose $\hat{\varphi} \in \mathcal{A}$ is optimal for the primal problem (2.6)).

Let $\left(\hat{p}_{1}(t), \hat{q}_{1}(t), \hat{r}_{1}(t, \zeta)\right)$ be the associated adjoint processes, solution of the constrained BSDE (2.13) $-(2.14)$. Define

$$
\hat{\theta}_{0}(t)=\frac{\hat{q}_{1}(t)}{\hat{p}_{1}\left(t^{-}\right)}, \quad \hat{\theta}_{1}(t, \zeta)=\frac{\hat{r}_{1}(t, \zeta)}{\hat{p}_{1}\left(t^{-}\right)}
$$

Suppose

$$
E\left[\int_{0}^{T}\left\{\hat{\theta}_{0}^{2}(t)+\int_{\mathbb{R}} \hat{\theta}_{1}^{2}(t, \zeta) \nu(d \zeta)\right\} d t\right]<\infty ; \quad \hat{\theta}_{1}>-1 .
$$

Then $\hat{\theta}=\left(\hat{\theta}_{0}, \hat{\theta}_{1}\right) \in \Theta$ is optimal for the dual problem (2.11) with initial value $y=\hat{p}_{1}(0)$. Moreover, with $y=\hat{p}_{1}(0)$,

$$
G_{\hat{\theta}}^{y}(t)=\hat{p}_{1}(t) ; \quad t \in[0, T]
$$

In particular

$$
G_{\hat{\theta}}^{y}(T)=U^{\prime}\left(X_{\hat{\varphi}}^{x}(T)\right) .
$$

b) Conversely, suppose $\hat{\theta}=\left(\hat{\theta}_{0}, \hat{\theta}_{1}\right) \in \Theta$ is optimal for the dual problem (2.11). Let $\left(\hat{p}_{2}(t), \hat{q}_{2}(t), \hat{r}_{2}(t, \zeta)\right)$ be the associated adjoint processes, solution of the BSDE (2.18) with the constraint (2.20). Suppose the portfolio

$$
\hat{\varphi}(t):=\frac{K\left(\hat{q}_{2}, \hat{r}_{2}\right)(t)}{S\left(t^{-}\right)}
$$

is admissible. Then $\hat{\varphi}$ is an optimal portfolio for the primal problem (2.6) with initial value $x=\hat{p}_{2}(0)$. Moreover, with $x=\hat{p}_{2}(0)$,

$$
X_{\hat{\varphi}}^{x}(t)=\hat{p}_{2}(t) ; \quad t \in[0, T] .
$$

In particular

$$
X_{\hat{\varphi}}^{x}(T)=-V^{\prime}\left(G_{\hat{\theta}}^{y}(T)\right)
$$

Proof. a) Suppose $\hat{\varphi}$ is optimal for problem (2.6) with initial value $x$. Then, by Proposition 2.3, the adjoint processes $\hat{p}_{1}(t), \hat{q}_{1}(t), \hat{r}_{1}(t, \zeta)$ for Problem (2.6) satisfy (2.13)-(2.14). Consider the process $\hat{\theta}(t)$ defined in (2.34) and suppose (2.35) holds. Then $\hat{\theta} \in \Theta$ and (2.13) can be written

$$
\left\{\begin{array}{l}
d \hat{p}_{1}(t)=\hat{p}_{1}\left(t^{-}\right)\left[\hat{\theta}_{0}(t) d B(t)+\int_{\mathbb{R}} \hat{\theta}_{1}(t, \zeta) \tilde{N}(d t, d \zeta)\right] \\
\hat{p}_{1}(T)=U^{\prime}\left(X_{\hat{\varphi}}^{x}(T)\right) .
\end{array}\right.
$$


Therefore $\hat{p}_{1}(t) \equiv G_{\hat{\theta}}^{y}(t)($ see (2.8) $)$ if we put $y:=\hat{p}_{1}(0)>0$, and we have, by (1.7)

$$
U^{\prime}\left(X_{\hat{\varphi}}^{x}(T)\right)=G_{\tilde{\theta}}^{y}(T) \text {, i.e. } X_{\hat{\varphi}}^{x}(T)=-V^{\prime}\left(G_{\tilde{\theta}}^{y}(T)\right) .
$$

Now define

$$
\hat{p}_{2}(t):=X_{\hat{\varphi}}^{x}(t), \hat{q}_{2}(t):=\hat{\varphi}(t) \sigma(t) S\left(t^{-}\right) \text {and } \hat{r}_{2}(t, \zeta):=\hat{\varphi}(t) \gamma(t, \zeta) S\left(t^{-}\right) .
$$

Then $\left(\hat{p}_{2}, \hat{q}_{2}, \hat{r}_{2}\right)$ satisfy the conditions of Proposition 2.6 which imply that $\hat{\theta}$ is optimal for problem (2.11).

b) Suppose $\hat{\theta} \in \Theta$ is optimal for problem (2.11) with initial value $y$. Let $\hat{p}_{2}(t), \hat{q}_{2}(t), \hat{r}_{2}(t, \cdot)$ be the associated adjoint processes, solution of the BSDE (2.18) with the constraint (2.20). Then they satisfy the equation

$$
\left\{\begin{array}{l}
d \hat{p}_{2}(t)=K\left(\hat{q}_{2}, \hat{r}_{2}\right)(t)\left[b(t) d t+\sigma(t) d B(t)+\int_{\mathbb{R}} \gamma(t, \zeta) \tilde{N}(d t, d \zeta)\right] \\
\hat{p}_{2}(T)=-V^{\prime}\left(G_{\hat{\theta}}(T)\right) .
\end{array}\right.
$$

Define

$$
\tilde{\varphi}(t):=\hat{\varphi}(t):=\frac{K\left(\hat{q}_{2}, \hat{r}_{2}\right)(t)}{S\left(t^{-}\right)},
$$

and assume $\tilde{\varphi}(t)$ is admissible. Then $\hat{p}_{2}(t) \equiv X_{\tilde{\varphi}}^{x}(t)$ for $x=\hat{p}_{2}(0)$. In particular

$$
X_{\tilde{\varphi}}^{x}(T)=-V^{\prime}\left(G_{\tilde{\theta}}^{y}(T)\right) \text {, i.e. } G_{\tilde{\theta}}^{y}(T)=U^{\prime}\left(X_{\tilde{\varphi}}^{x}(T)\right) .
$$

Therefore $G_{\hat{\theta}}^{y}(t)=G_{\hat{\theta}}(t)$ satisfies the equation

$$
\left\{\begin{array}{l}
d G_{\hat{\theta}}(t)=G_{\hat{\theta}}\left(t^{-}\right)\left[\hat{\theta}_{0}(t) d B(t)+\int_{\mathbb{R}} \hat{\theta}_{1}(t, \zeta) \tilde{N}(d t, d \zeta)\right] ; 0 \leq t \leq T \\
G_{\hat{\theta}}(T)=U^{\prime}\left(X_{\tilde{\varphi}}^{x}(T)\right) .
\end{array}\right.
$$

Define now

$$
p_{1}(t):=G_{\hat{\theta}}(t), q_{1}(t):=G_{\hat{\theta}}(t) \hat{\theta}_{0}(t), r_{1}(t, \zeta):=G_{\hat{\theta}}(t) \hat{\theta}_{1}(t, \zeta) .
$$

Then by (2.47) $\left(p_{1}, q_{1}, r_{1}\right)$ solves the BSDE

$$
\left\{\begin{array}{l}
d p_{1}(t)=q_{1}(t) d B(t)+\int_{\mathbb{R}} r_{1}(t, \zeta) \tilde{N}(d t, d \zeta) ; 0 \leq t \leq T \\
p_{1}(T)=U^{\prime}\left(X_{\tilde{\varphi}}^{x}(T)\right) .
\end{array}\right.
$$

Moreover, since $\hat{\theta} \in \Theta$, it satisfies (2.10), that is

$$
b(t)+\sigma(t) \hat{\theta}_{0}(t)+\int_{\mathbb{R}} \gamma(t, \zeta) \hat{\theta}_{1}(t, \zeta) \nu(d \zeta)=0 ; 0 \leq t \leq T
$$


i.e., $\left(p_{1}, q_{1}, r_{1}\right)$ satisfies the equation

$$
b(t)+\sigma(t) \frac{q_{1}(t)}{p_{1}(t)}+\int_{\mathbb{R}} \gamma(t, \zeta) \frac{r_{1}(t, \zeta)}{p_{1}(t)} \nu(d \zeta)=0 ; 0 \leq t \leq T .
$$

It follows from Proposition 2.3 that $\hat{\varphi}:=\tilde{\varphi}$ is an optimal portfolio for problem (2.6) with initial value $x=\hat{p}_{2}(0)$.

Remark 2.9 Conditions of the above theorem have to be verified in each specific case. They hold for examples in in Examples [2.1 and [3.1. Note that the integrability condition in (2.35) hold whenever the utility function $U$ satisfies the condition

$$
U^{\prime} \text { is bounded and bounded away from } 0 .
$$

Indeed this implies that $p_{1}(t)$ which is equal to $E\left[U^{\prime}\left(X_{\hat{\varphi}}^{x}(T)\right) \mid \mathcal{F}_{t}\right]$ is bounded away from 0 and that $\left(q_{1}, r_{1}\right)$ belongs to $H^{2} \times H_{\nu}$. Therefore $\frac{1}{p_{1}}$ is bounded and $\left(\frac{q_{1}}{p_{1}}, \frac{r_{1}}{p_{1}}\right)$ belong to $H^{2} \times H_{\nu}$. Condition (2.52) does not hold a priori for the most commonly studied utility functions, e.g. the logarithmic or the power functions, but any given utility function can be perturbed slightly such that it holds, simply by modifying it arbitrary near 0 or arbitrary near infinity, if necessary.

Example 2.1 As an illustration of Theorem 2.8 let us apply it to the situation when $\sigma=0$, $\gamma(t, \zeta)=\gamma(t, 1)>0$ and $N(t)$ is the Poisson process with intensity $\lambda>0$. Then $\nu(d \zeta)=$ $\lambda \delta_{1}(d \zeta)$, where $\delta_{1}$ is Dirac measure at 1 , and hence

$$
\int_{\mathbb{R}} \gamma(t, \zeta) \tilde{N}(d t, d \zeta)=\gamma(t, 1)(d N(t)-\lambda d t):=\gamma(t, 1) d \tilde{N}(t)
$$

and (2.1) and (2.3) become, respectively,

$$
d S(t)=S\left(t^{-}\right)[b(t) d t+\gamma(t, 1) d \tilde{N}(t)] ; S(0)>0
$$

$\{$ eq3.20a\}

and

$$
d X(t)=\varphi(t) S\left(t^{-}\right)[b(t) d t+\gamma(t, 1) d \tilde{N}(t)] ; X(0)=x>0 .
$$

Assume that $b(t)$ and $\gamma(t, 1)$ are bounded predictable processes and that there exists a constant $C<1$ such that

$$
\frac{|b(t)|}{\lambda|\gamma(t, 1)|} \leq C ; 0 \leq t \leq T \text {. }
$$

Then $\Theta$ has just one element $\theta_{1}(t, 1)$, given by

$$
\theta_{1}(t, 1)=-\frac{b(t)}{\lambda \gamma(t, 1)}
$$


and hence by (2.10) and the Itô formula,

$$
\begin{aligned}
G_{\theta_{1}}^{y}(t) & =y \exp \left(\int_{0}^{t} \ln \left(1-\frac{b(s)}{\lambda \gamma(s, 1)}\right) d \tilde{N}(s)\right. \\
& \left.+\lambda \int_{0}^{t}\left\{\ln \left(1-\frac{b(s)}{\lambda \gamma(s, 1)}\right)+\frac{b(s)}{\lambda \gamma(s, 1)}\right\} d s\right) ; 0 \leq t \leq T .
\end{aligned}
$$

By Theorem $2.8 \mathrm{~b})$ we get that

$$
\hat{\varphi}(t):=\frac{\hat{r}_{2}(t, 1)}{\gamma(t, 1) S\left(t^{-}\right)}
$$

is an optimal portfolio for the primal problem (2.8), where $\left(\hat{p}_{2}(t), \hat{r}_{2}(t, 1)\right)$ solves the BSDE (2.18), which in our case gets the form

$$
\left\{\begin{array}{l}
d \hat{p}_{2}(t)=\frac{\hat{r}_{2}(t, 1)}{\gamma(t, 1)} b(t) d t+\hat{r}_{2}(t, 1) d \tilde{N}(t) ; 0 \leq t \leq T \\
\hat{p}_{2}(T)=-V^{\prime}\left(G_{\theta_{1}}^{y}(T)\right) .
\end{array}\right.
$$

To solve this BSDE we try a solution of the form

$$
\hat{r}_{2}(t, 1)=\hat{p}_{2}(t) \psi(t)
$$

for some predictable process $\psi$, and get the solution

$$
\begin{aligned}
\hat{p}_{2}(t) & =\hat{p}_{2}(0) \exp \left(\int_{0}^{t} \ln (1+\psi(s)) d \tilde{N}(s)\right. \\
& \left.+\int_{0}^{t}\left\{\lambda(\ln (1+\psi(s))-\psi(s))+\frac{b(s)}{\gamma(s, 1)} \psi(s)\right\} d s\right) .
\end{aligned}
$$

In particular, if $U(x)=\ln x$, then $V(y)=-\ln y-1$ and $V^{\prime}(y)=-\frac{1}{y}$. Hence (2.60) implies that

$$
\begin{aligned}
\hat{p}_{2}(0) & \exp \left(\int_{0}^{T} \ln (1+\psi(s)) d \tilde{N}(s)\right. \\
& +\int_{0}^{T}\left\{\lambda(\ln (1+\psi(s))-\psi(s))+\frac{b(s)}{\gamma(s, 1)} \psi(s)\right\} d t \\
& =\frac{1}{y} \exp \left(-\int_{0}^{T} \ln \left(1-\frac{b(s)}{\lambda \gamma(s, 1)}\right) d \tilde{N}(s)\right. \\
& \left.-\lambda \int_{0}^{T}\left\{\ln \left(1-\frac{b(s)}{\lambda \gamma(s, 1)}\right)+\frac{b(s)}{\lambda \gamma(s, 1)}\right\} d s\right) .
\end{aligned}
$$

Choose

$$
\hat{p}_{2}(0)=\frac{1}{y}
$$


and choose $\psi(s)$ such that the $d \tilde{N}$-integrals of (2.63) coincide, i.e.

$$
\ln (1+\psi(s))=-\ln \left(1-\frac{b(s)}{\lambda \gamma(s, 1)}\right)
$$

i.e.

$$
\psi(s)=\frac{b(s)}{\lambda \gamma(s, 1)-b(s)} .
$$

Then we see that also the $d s$-integrals coincide, i.e.

$$
\lambda(\ln (1+\psi(s))-\psi(s))+\frac{b(s)}{\gamma(s, 1)} \psi(s)=-\lambda\left(\ln \left(1-\frac{b(s)}{\lambda \gamma(s, 1)}\right)+\frac{b(s)}{\lambda \gamma(s, 1)}\right) .
$$

Hence, with this choice of $\psi$, we see that $d\left(\hat{p}_{2}(t)\right)=d\left(\frac{1}{G_{\theta_{1}}^{y}}\right)(t)$. The process

$$
\hat{p}_{2}=\frac{1}{G_{\theta_{1}}^{y}} ; \hat{r}_{2}=\hat{p}_{2} \psi
$$

with $\psi$ given by (2.65) solves BSDE (2.60). Moreover (2.20) holds trivially. We conclude by (2.59) that the optimal portfolio $\hat{\varphi}(t)$ for problem (2.8) with $U(x)=\ln x$ is

$$
\hat{\varphi}(t)=\frac{\hat{p}_{2}(t) b(t)}{\gamma(t, 1) S\left(t^{-}\right)(\lambda \gamma(t, 1)-b(t))}
$$

which means that the optimal fraction $\hat{\pi}(t)$ to be placed in the risky asset is, using (2.39)

$$
\hat{\pi}(t)=\frac{\hat{\varphi}(t) S\left(t^{-}\right)}{X_{\hat{\varphi}}(t)}=\frac{b(t)}{\gamma(t, 1)(\lambda \gamma(t, 1)-b(t))} .
$$

Remark 2.10 To check that $\hat{\varphi}$ is admissible, we have to verify that (2.4) and (2.5) hold for $\varphi=\hat{\varphi}$. To this end, we see that condition (2.56) suffices.

\section{Robust duality}

In this section we extend our study to a robust optimal portfolio problem and its dual.

\subsection{Model uncertainty setup}

To get a representation of model uncertainty, we consider a family of probability measures $R=R^{\kappa} \sim P$, with Radon-Nikodym derivative on $\mathcal{F}_{t}$ given by

$$
\frac{d\left(R^{\kappa} \mid \mathcal{F}_{t}\right)}{d\left(P \mid \mathcal{F}_{t}\right)}=Z_{t}^{\kappa}
$$


where, for $0 \leq t \leq T, Z_{t}^{\kappa}$ is a martingale of the form

$$
d Z_{t}^{\kappa}=Z_{t^{-}}^{\kappa}\left[\kappa_{0}(t) d B_{t}+\int_{\mathbb{R}} \kappa_{1}(t, \zeta) \tilde{N}(d t, d \zeta)\right] ; \quad Z_{0}^{\kappa}=1 .
$$

Let $\mathbb{K}$ denote a given set of admissible scenario controls $\kappa=\left(\kappa_{0}, \kappa_{1}\right), \mathcal{F}_{t}$-predictable, s.t. $\kappa_{1}(t, z) \geq-1+\epsilon$, and $E\left[\int_{0}^{T}\left\{\left|\kappa_{0}^{2}(t)\right|+\int_{\mathbb{R}} \kappa_{1}^{2}(t, z) \nu(d z)\right\} d t\right]<\infty$.

By the Girsanov theorem, using the measure $R^{\kappa}$ instead of the original measure $P$ in the computations involving the price process $S(t)$, is equivalent to using the original measure $P$ in the computations involving the perturbed price process $S_{\mu}(t)$ instead of $P(t)$, where $S_{\mu}(t)$ is given by

$$
\left\{\begin{array}{l}
d S_{\mu}(t)=S_{\mu}\left(t^{-}\right)\left[(b(t)+\mu(t)) d t+\sigma(t) d B(t)+\int_{\mathbb{R}} \gamma(t, \zeta) \tilde{N}(d t, d \zeta)\right] \\
S_{\mu}(0)>0
\end{array}\right.
$$

with

$$
\mu(t)=-\sigma(t) \kappa_{0}(t)-\int_{\mathbb{R}} \gamma(t, \zeta) \kappa_{1}(t, \zeta) \nu(d \zeta) d t
$$

Accordingly, we now replace the price process $S(t)$ in (2.1) by the perturbed process

$$
\left\{\begin{array}{l}
d S_{\mu}(t)=S_{\mu}\left(t^{-}\right)\left[(b(t)+\mu(t)) d t+\sigma(t) d B(t)+\int_{\mathbb{R}} \gamma(t, \zeta) \tilde{N}(d t, d \zeta)\right] ; 0 \leq t \leq T \\
S_{\mu}(0)>0
\end{array}\right.
$$

for some perturbation process $\mu(t)$, assumed to be predictable and satisfy

$$
E\left[\int_{0}^{T}|\mu(t)| d t\right]<\infty .
$$

Let $\mathbb{M}$ denote this set of perturbation processes $\mu$. Let $X=X_{\varphi, \mu}^{x}$ be the corresponding wealth process given by

$$
\left\{\begin{array}{l}
d X(t)=\varphi(t) S_{\mu}\left(t^{-}\right)\left[(b(t)+\mu(t)) d t+\sigma(t) d B(t)+\int_{\mathbb{R}} \gamma(t, \zeta) \tilde{N}(d t, d \zeta)\right] ; 0 \leq t \leq T \\
X(0)=x>0
\end{array}\right.
$$

where $\varphi$ is an admissible portfolio, that is it belongs to the set $\mathcal{A}$ of $\mathbb{F}$-predictable processes such that

$$
\left\{\begin{array}{l}
(2.4) \text { and (2.5) hold, } \\
E\left[\int_{0}^{T} \varphi(t)^{2} S_{\mu}(t)^{2}\left\{(b(t)+\mu(t))^{2}+\sigma^{2}(t)+\int_{\mathbb{R}} \gamma^{2}(t, \zeta) \nu(d \zeta)\right\} d t\right]<\infty \\
X_{\varphi, \mu}(t)>0 \text { for all } t \in[0, T] \text { a.s. }
\end{array}\right.
$$

for all $\mu \in \mathbb{M}$. 


\subsection{The robust primal and dual problems}

Let $\rho: \mathbb{R} \rightarrow \mathbb{R}$ be a convex penalty function, assumed to be $\mathcal{C}^{1}$, and $U$ a utility function as in Section 1. We assume that $\rho(\mu)$ has a minimum at $\mu=0$ and that $\rho(0)=0$. Then $\rho(\mu)$ can be interpreted as a penalization for choosing $\mu \neq 0$.

Definition 3.1 The robust primal problem is, for given $x>0$, to find $(\hat{\varphi}, \hat{\mu}) \in \mathcal{A} \times \mathbb{M}$ such that

$$
\inf _{\mu \in \mathbb{M}} \sup _{\varphi \in \mathcal{A}} I(\varphi, \mu)=I(\hat{\varphi}, \hat{\mu})=\sup _{\varphi \in \mathcal{A}} \inf _{\mu \in \mathbb{M}} I(\varphi, \mu),
$$

where

$$
I(\varphi, \mu)=E\left[U\left(X_{\varphi, \mu}^{x}(T)\right)+\int_{0}^{T} \rho(\mu(t)) d t\right] .
$$

The problem (3.54) is a stochastic differential game. To handle this, we use an extension of the maximum principle to games, as presented in, e.g., [15]. We obtain the following characterization of a solution (saddle point) of (3.54):

Proposition 3.2 (Robust primal problem and associated constrained FBSDE) A pair $(\hat{\varphi}, \hat{\mu}) \in \mathcal{A} \times \mathbb{M}$ is a solution of the robust primal problem (3.54) if and only if the solution $X(t),\left(p_{1}, q_{1}, r_{1}\right)$ of the FBSDE consisting of the SDE (3.4) and the BSDE

$$
\left\{\begin{array}{l}
d p_{1}(t)=q_{1}(t) d B(t)+\int_{\mathbb{R}} r_{1}(t, \zeta) \tilde{N}(d t, d \zeta) ; 0 \leq t \leq T \\
p_{1}(T)=U^{\prime}\left(X_{\hat{\varphi}, \hat{\mu}}^{x}(T)\right)
\end{array}\right.
$$

satisfies

$$
\begin{gathered}
(b(t)+\hat{\mu}(t)) p_{1}(t)+\sigma(t) q_{1}(t)+\int_{\mathbb{R}} \gamma(t, \zeta) r_{1}(t, \zeta) \nu(d \zeta)=0 ; t \in[0, T] \\
\rho^{\prime}(\hat{\mu}(t))+\hat{\varphi}(t) S_{\hat{\mu}}\left(t^{-}\right) p_{1}(t)=0 ; t \in[0, T] .
\end{gathered}
$$

Proof. Define the Hamiltonian by

$$
H_{1}(t, x, \varphi, \mu, p, q, r)=\rho(\mu)+\varphi S_{\mu}\left(t^{-}\right)\left[(b(t)+\mu) p+\sigma(t) q+\int_{\mathbb{R}} \gamma(t, \zeta) r(\zeta) \nu(d \zeta)\right] .
$$

The associated BSDE for the adjoint processes $\left(p_{1}, q_{1}, r_{1}\right)$ is (3.8).

The first order conditions for a maximum point $\hat{\varphi}$ and a minimum point $\hat{\mu}$, respectively, for the Hamiltonian are given by (3.9) and (3.10). Since $H_{1}$ is concave with respect to $\varphi$ and convex with respect to $\mu$, these first order conditions are also sufficient for $\hat{\varphi}$ and $\hat{\mu}$ to be a maximum point and a minimum point, respectively.

We now study a dual formulation of the robust primal problem (3.54). Let now $\mathcal{M}$ be the family of positive measures $Q=Q_{\theta, \mu}$ of the form

$$
d Q_{\theta, \mu}(\omega)=G_{\theta, \mu}(T) d P(\omega) \text { on } \mathcal{F}_{T},
$$

$\{$ eq2.5a $\}$ 
where $G(t)=G_{\theta, \mu}^{y}(t)$ is given by

$$
\left\{\begin{array}{l}
d G(t)=G\left(t^{-}\right)\left[\theta_{0}(t) d B(t)+\int_{\mathbb{R}} \theta_{1}(t, \zeta) \tilde{N}(d t, d \zeta)\right] ; 0 \leq t \leq T \\
G(0)=y>0
\end{array}\right.
$$

and $(\theta, \mu)$ is such that $\mu \in \mathbb{M}$ and $\theta=\left(\theta_{0}, \theta_{1}\right)$ is a predictable processes satisfying (2.9) and

$$
b(t)+\mu(t)+\sigma(t) \theta_{0}(t)+\int_{\mathbb{R}} \gamma(t, \zeta) \theta_{1}(t, \zeta) \nu(d \zeta)=0 ; t \in[0, T] .
$$

We let $\Lambda$ denote the set of such processes $(\theta, \mu)$. If $y=1$, then the measure $Q_{\theta, \mu}$ is an ELMM for the perturbed price process $S_{\mu}$ in (3.3).

Definition 3.3 The robust dual problem is for given $y>0$, to find $(\tilde{\theta}, \tilde{\mu}) \in \Lambda$ such that

$$
\sup _{(\theta, \mu) \in \Lambda} J(\theta, \mu)=J(\tilde{\theta}, \tilde{\mu})
$$

where

$$
J(\theta, \mu)=E\left[-V\left(G_{\theta, \mu}^{y}(T)\right)-\int_{0}^{T} \rho(\mu(t)) d t\right]
$$

and $V$ is the convex conjugate function of $U$, as in Section 1 .

Proposition 3.4 (Robust dual problem and its associated constrained FBSDE.) A pair $(\tilde{\theta}, \tilde{\mu}) \in \Lambda$ is a solution of the robust dual problem (3.55) -(3.16) if and only the solution $G(t),\left(p_{2}, q_{2}, r_{2}\right)$ of the FBSDE consisting of the FSDE (3.13) and the BSDE

$$
\left\{\begin{array}{l}
d p_{2}(t)=K\left(q_{2}, r_{2}\right)(t)[b(t)+\tilde{\mu}(t)] d t+q_{2}(t) d B(t)+\int_{\mathbb{R}} r_{2}(t, \zeta) \tilde{N}(d t, d \zeta) ; t \in[0, T] \\
p_{2}(T)=-V^{\prime}\left(G_{\tilde{\theta}, \tilde{\mu}}^{y}(T)\right)
\end{array}\right.
$$

with $K(q, r)(t)$ defined as in (2.19), satisfies the two equations

$$
\begin{gathered}
G_{\tilde{\theta}, \tilde{\mu}}^{y}(t) q_{2}(t)+\rho^{\prime}(\tilde{\mu}(t)) \sigma(t)=0, \\
G_{\tilde{\theta}, \tilde{\mu}}^{y}(t) r_{2}(t, \zeta)+\rho^{\prime}(\tilde{\mu}(t)) \gamma(t, \zeta)=0 .
\end{gathered}
$$

Proof. We proceed as in the proof of Proposition 2.6. The Hamiltonian for the constrained stochastic control problem (3.55) is

$$
\begin{aligned}
& H_{2}^{L}\left(t, g, \theta_{0}, \theta_{1}, \mu, p, q, r\right) \\
& :=-\rho(\mu)+g \theta_{0} q+g \int_{\mathbb{R}} \theta_{1}(\zeta) r(\zeta) \nu(d \zeta)+L(t)\left(b(t)+\mu(t)+\sigma(t) \theta_{0}+\int_{\mathbb{R}} \gamma(t, \zeta) \theta_{1}(\zeta) \nu(d \zeta)\right),
\end{aligned}
$$


where $L(t)$ is the Lagrange multiplier process.

The first order conditions for a maximum point $(\tilde{\theta}, \tilde{\mu})$ for $H_{2}^{L}$ are $\nabla_{\theta} H_{2}^{L}=0$ and $\left(\frac{\partial H_{2}^{L}}{\partial \mu}\right)=0$ which reduce to (3.18)$-(\underline{3.19})$. Then, as in (2.27) we see that the corresponding BSDE for the adjoint processes $\left(p_{2}, q_{2}, r_{2}\right)$ is given by (3.17).

Since $H_{2}$ is concave w.r.t. $\mu$ and $\theta$, these necessary optimality conditions are also sufficient.

\subsection{Relations between robust primal and robust dual problems}

We now use the characterizations above of the solutions $(\hat{\varphi}, \hat{\mu}) \in \mathcal{A} \times \mathbb{M}$ and $(\tilde{\theta}, \tilde{\mu}) \in \Lambda$ of the robust primal and the robust dual problem, respectively, to find the relations between them.

Theorem 3.5 (i) From robust primal to robust dual.

Assume $(\hat{\varphi}, \hat{\mu}) \in \mathcal{A} \times \mathbb{M}$ is a solution of the robust primal problem and let $\left(p_{1}, q_{1}, r_{1}\right)$ be the associated adjoint processes solution of the FBSDE (3.4) \&6 (3.8) and satisfying (3.9) -(3.10). Define

$$
\begin{aligned}
& \tilde{\mu}:=\hat{\mu} \\
& \tilde{\theta}_{0}(t):=\frac{q_{1}(t)}{p_{1}(t)} ; \quad \tilde{\theta}_{1}(t, \zeta)=\frac{r_{1}(t, \zeta)}{p_{1}(t)}
\end{aligned}
$$

and suppose they satisfy (2.9). Then, they are optimal for the dual problem with initial value $y=p_{1}(0)$. Moreover

$$
p_{1}(t)=G_{\tilde{\theta}, \tilde{\mu}}(t) ; t \in[0, T] .
$$

In particular,

$$
U^{\prime}\left(X_{\hat{\varphi}, \hat{\mu}}(T)\right)=G_{\tilde{\theta}, \tilde{\mu}}(T) .
$$

(ii) From robust dual to robust primal Let $(\tilde{\theta}, \tilde{\mu}) \in \Lambda$ be optimal for the robust dual problem (3.55)-(3.16) and let $\left(p_{2}, q_{2}, r_{2}\right)$ be the associated adjoint processes satisfying (3.17) with the constraints (3.19) and (3.18). Define

$$
\begin{aligned}
& \hat{\mu}:=\tilde{\mu} \\
& \hat{\varphi}(t):=\frac{K\left(q_{2}, r_{2}\right)(t)}{S_{\hat{\mu}}\left(t^{-}\right)} ; t \in[0, T] .
\end{aligned}
$$

Assume that $\hat{\varphi} \in \mathcal{A}$. Then $(\hat{\mu}, \hat{\varphi})$ are optimal for primal problem with initial value $x=p_{2}(0)$. Moreover,

$$
p_{2}(t)=X_{\hat{\varphi}, \hat{\mu}}(t) \quad t \in[0, T] .
$$

$\{$ eq4.42a $\}$

In particular

$$
-V^{\prime}\left(G_{\tilde{\theta}}(T)\right)=X_{\hat{\varphi}, \hat{\mu}}(T) .
$$


Proof. (i) Let $(\hat{\varphi}, \hat{\mu}) \in \mathcal{A} \times \mathbb{M}$ is a solution of the robust primal problem and let $\left(p_{1}, q_{1}, r_{1}\right)$ be as in Proposition 3.2. i.e. assume that $\left(p_{1}, q_{1}, r_{1}\right)$ solves the FBSDE (3.4) and (3.8) and satisfies (3.9) $-(3.10)$.

We want to find the solution $(\tilde{\theta}, \tilde{\mu}) \in \Lambda$ of the robust dual problem. By Proposition 3.4 this means that we must find a solution $\left(p_{2}, q_{2}, r_{2}\right)$ of the FBSDE (3.13) and (3.17) which satisfies (3.19) $-(3.18)$. To this end, choose $\tilde{\mu}, \tilde{\theta}_{0}, \tilde{\theta}_{1}$ given in (3.21)-(3.22). Then by (3.9) we have

$$
b(t)+\tilde{\mu}(t)+\sigma(t) \tilde{\theta}_{0}(t)+\int_{\mathbb{R}} \gamma(t, \zeta) \tilde{\theta}_{1}(t, \zeta) \nu(d \zeta)=0 .
$$

Assume that (2.9) holds. Then $(\tilde{\mu}, \tilde{\theta}) \in \Lambda$. Substituting (3.22) into (3.8), we obtain

$$
\left\{\begin{array}{l}
d p_{1}(t)=p_{1}\left(t^{-}\right)\left[\tilde{\theta}_{0}(t) d B(t)+\int_{\mathbb{R}} \tilde{\theta}_{1}(t, \zeta) \tilde{N}(d t, d \zeta)\right] ; t \in[0, T] \\
p_{1}(T)=U^{\prime}\left(X_{\hat{\varphi}, \hat{\mu}}(T)\right) .
\end{array}\right.
$$

Comparing with (3.13) we see that

$$
\frac{d G_{\tilde{\theta}, \tilde{\mu}}(t)}{G_{\tilde{\theta}, \tilde{\mu}}(t)}=\frac{d p_{1}(t)}{p_{1}(t)}
$$

and hence, for $y=G_{\tilde{\theta}, \tilde{\mu}}(0)=p_{1}(0)>0$ we get (3.23) and (3.24). Define

$$
p_{2}(t):=X_{\hat{\varphi}, \hat{\mu}}(t), q_{2}(t):=\hat{\varphi}(t) \sigma(t) S_{\hat{\mu}}\left(t^{-}\right), r_{2}(t, \zeta):=\hat{\varphi}(t) \gamma(t, \zeta) S_{\hat{\mu}}\left(t^{-}\right) .
$$

Then by (3.4) and (3.24), combined with (1.7),

$$
\left\{\begin{array}{l}
d p_{2}(t)=\hat{\varphi}(t) S_{\hat{\mu}}\left(t^{-}\right)\left[(b(t)+\hat{\mu}(t)) d t+\sigma(t) d B(t)+\int_{\mathbb{R}} \gamma(t, \zeta) \tilde{N}(d t, d \zeta)\right] \\
\quad=K\left(q_{2}, r_{2}\right)(t)[b(t)+\hat{\mu}(t)] d t+q_{2}(t) d B(t)+\int_{\mathbb{R}} r_{2}(t, \zeta) \tilde{N}(d t, d \zeta) ; 0 \leq t \leq T \\
p_{2}(T)=X_{\hat{\varphi}, \hat{\mu}}(T)=-V^{\prime}\left(G_{\tilde{\theta}, \tilde{\mu}}(T)\right) .
\end{array}\right.
$$

Hence $\left(p_{2}, q_{2}, r_{2}\right)$ solves the BSDE (3.17), as requested. It remains to verify that (3.19) and (3.18) hold: By (3.31) we have

$$
-q_{2}(t) \gamma(t, \zeta)+\sigma(t) r_{2}(t, \zeta)=\sigma(t)\left[-\hat{\varphi}(t) S_{\hat{\mu}}\left(t^{-}\right) \gamma(t, \zeta)+\hat{\varphi}(t) S_{\hat{\mu}}\left(t^{-}\right) \gamma(t, \zeta)\right]=0,
$$

which is (3.19). By (3.21), (3.23), (3.31) and (3.10),

$$
\rho^{\prime}(\tilde{\mu})+G_{\tilde{\theta}, \tilde{\mu}}(t) q_{2}(t)=\rho^{\prime}(\hat{\mu})+p_{1}(t) \hat{\varphi}(t) \sigma(t) S_{\hat{\mu}}\left(t^{-}\right)=0,
$$

which is (3.18).

(ii) Next, assume that $(\tilde{\theta}, \tilde{\mu}) \in \Lambda$ is optimal for the robust dual problem (3.55)-(3.16) and let $\left(p_{2}, q_{2}, r_{2}\right)$ be as in Proposition 3.4. We will find $(\hat{\varphi}, \hat{\mu}) \in \mathcal{A} \times \mathbb{M}$ and $\left(p_{1}, q_{1}, r_{1}\right)$ satisfying 
Proposition 3.2. Choose $\hat{\mu}$ and $\hat{\varphi}$ given in (3.25)-(3.26) and assume that $\hat{\varphi}$ is admissible. Then by (3.17) and (3.19)

$$
\left\{\begin{array}{l}
d p_{2}(t)=\hat{\varphi}(t) S_{\hat{\mu}}\left(t^{-}\right)\left[(b(t)+\hat{\mu}(t) \sigma(t)) d t+\sigma(t) d B(t)+\int_{\mathbb{R}} \gamma(t, \zeta) \tilde{N}(d t, d \zeta)\right] ; 0 \leq t \leq T \\
p_{2}(T)=-V^{\prime}\left(G_{\tilde{\theta}, \tilde{\mu}}(T)\right) .
\end{array}\right.
$$

Hence, with $x=p_{2}(0)>0$, (3.27) holds. In particular

$$
X_{\hat{\varphi}, \hat{\mu}}(T)=p_{2}(T)=-V^{\prime}\left(G_{\tilde{\theta}, \tilde{\mu}}(T)\right) \text {, i.e. } G_{\tilde{\theta}, \tilde{\mu}}(T)=U^{\prime}\left(X_{\hat{\varphi}, \hat{\mu}}(T)\right) .
$$

We now verify that with $\varphi=\hat{\varphi}, \mu=\hat{\mu}$, and $p_{1}, q_{1}, r_{1}$ defined by

$$
p_{1}(t):=G_{\tilde{\theta}, \tilde{\mu}}(t), q_{1}(t):=G_{\tilde{\theta}, \tilde{\mu}}(t) \tilde{\theta}_{0}(t), r_{1}(t, \zeta):=G_{\tilde{\theta}, \tilde{\mu}}(t) \tilde{\theta}_{1}(t, \zeta),
$$

all the conditions of Proposition 3.2 hold: By (3.13) and (3.33),

$$
\left\{\begin{array}{l}
d p_{1}(t)=d G_{\tilde{\theta}, \tilde{\mu}}(t)=G_{\tilde{\theta}, \tilde{\mu}}\left(t^{-}\right)\left(\tilde{\theta}_{0}(t) d B(t)+\int_{\mathbb{R}} \tilde{\theta}_{1}(t, \zeta) \tilde{N}(d t, d \zeta)\right) \\
=q_{1}(t) d B(t)+\int_{\mathbb{R}} r_{1}(t, \zeta) \tilde{N}(d t, d \zeta) \quad ; 0 \leq t \leq T \\
p_{1}(T)=G_{\tilde{\theta}, \tilde{\mu}}(T)=U^{\prime}\left(X_{\hat{\varphi}, \hat{\mu}}(T)\right) .
\end{array}\right.
$$

Hence (3.8) holds. It remains to verify (3.9) and (3.10). By (3.34) and (3.14) for $\theta=\tilde{\theta}$, we get

$$
\begin{aligned}
&(b(t)+\hat{\mu}(t)) p_{1}(t)+\sigma(t) q_{1}(t)+\int_{\mathbb{R}} \gamma(t, \zeta) r_{1}(t, \zeta) \nu(d \zeta) \\
&=G_{\tilde{\theta}, \tilde{\mu}}(t)\left[b(t)+\hat{\mu}(t)+\sigma(t) \tilde{\theta}_{0}(t)+\int_{\mathbb{R}} \gamma(t, \zeta) \tilde{\theta}_{1}(t, \zeta) \nu(d \zeta)\right]=0
\end{aligned}
$$

which is (3.9). By (3.25), (3.26), (3.34) and (3.18) we get

$$
\rho^{\prime}(\hat{\mu}(t))+\hat{\varphi}(t) S_{\hat{\mu}}\left(t^{-}\right) \sigma(t) p_{1}(t)=\rho^{\prime}(\tilde{\mu}(t))+q_{2}(t) G_{\tilde{\theta}, \tilde{\mu}}(t)=0
$$

which is (3.10).

\subsection{Illustrating examples}

Example 3.1 We consider a robust version of the classical Merton type optimal portfolio problem: We assume that there exists a constant $C>0$ such that

$$
\frac{|b(t)|}{|\sigma(t)|} \leq C ; 0 \leq t \leq T
$$


We want to study

$$
\inf _{\mu \in \mathbb{M}} \sup _{\varphi \in \mathcal{A}} E\left[U\left(X_{\varphi, \mu}(T)\right)+\int_{0}^{T} \rho(\mu(t)) d t\right]
$$

in the case with no jumps $(N=\gamma=0, \sigma \neq 0)$. Then there is only one ELMM for the price process $S_{\mu}(t)$ for each given $\mu(t)$. So $\theta=\theta_{0}=-\frac{b(t)+\mu(t)}{\sigma(t)}$ and the corresponding robust dual problem simplifies to

$$
\sup _{\mu \in \mathbb{M}} E\left[-V\left(G_{\mu}(T)\right)-\int_{0}^{T} \rho(\mu(t)) d t\right]
$$

where

$$
d G_{\mu}(t)=-G_{\mu}\left(t^{-}\right) \frac{b(t)+\mu(t)}{\sigma(t)} d B_{t} ; 0 \leq t \leq T ; \quad G_{\mu}(0)=y>0 .
$$

The first order conditions for the Hamiltonian reduce to:

$$
\tilde{\mu}(t)=\left(\rho^{\prime}\right)^{-1}\left(-\frac{G_{\tilde{\mu}}(t) q_{2}(t)}{\sigma(t)}\right)
$$

which substituted into the adjoint BSDE equation gives:

$$
\left\{\begin{array}{l}
d p_{2}(t)=\frac{q_{2}(t)}{\sigma(t)}\left[b(t)+\left(\rho^{\prime}\right)^{-1}\left(-\frac{G_{\tilde{\mu}}(t) q_{2}(t)}{\sigma(t)}\right)\right] d t+q_{2}(t) d B_{t} ; \quad t \in[0, T] \\
p_{2}(T)=-V^{\prime}\left(G_{\tilde{\mu}}(T)\right) .
\end{array}\right.
$$

We get that $\tilde{\mu}$ is optimal for the robust dual problem if and only if there is a solution $\left(p_{2}, q_{2}, G_{\tilde{\mu}}\right)$ of the FBSDE consisting of (3.41) and (3.39) with the constraint (3.40). Hence, by Theorem 3.5 (ii), the optimal $\hat{\mu}$ for the primal robust problem is given by $\hat{\mu}:=\tilde{\mu}$, and the optimal portfolio is

$$
\hat{\varphi}(t)=\frac{K\left(q_{2}, r_{2}\right)(t)}{S_{\hat{\mu}}\left(t^{-}\right)}=\frac{q_{2}(t)}{\sigma(t) S_{\tilde{\mu}}\left(t^{-}\right)} ; t \in[0, T]
$$

Now assume that

$$
U(x)=\ln x \quad \text { and } \rho(x)=\frac{1}{2} x^{2}
$$

Then $V(y)=-\ln y-1$.

If $b(t)$ and $\sigma(t)$ are deterministic, we can solve (3.38) by dynamic programming, and we get

$$
\tilde{\mu}(t)=-\frac{b(t)}{2} ; t \in[0, T]
$$

In view of this, it is natural to guess that (3.44) is the optimal choice of $\mu$ also when $b(t)$ and $\sigma(t)$ are $\mathcal{F}_{t}$-adapted processes. To verify this we have to show that the system (3.39)-(3.41) 
is consistent. This system is now the following

$$
\begin{aligned}
G_{\tilde{\mu}}(t) & =y \exp \left(-\int_{0}^{t} \frac{b(s)}{2 \sigma(s)} d B(s)-\frac{1}{2}\left(\frac{b(s)}{2 \sigma(s)}\right)^{2} d s\right) \\
q_{2}(t) & =\frac{1}{G_{\tilde{\mu}}(t)} \cdot \frac{b(t)}{2 \sigma(t)} \\
d p_{2}(t) & =\frac{1}{G_{\tilde{\mu}}(t)}\left[\frac{b(t)}{2 \sigma(t)} d B(t)+\left(\frac{b(t)}{2 \sigma(t)}\right)^{2} d t\right] ; p_{2}(T)=\frac{1}{G_{\tilde{\mu}}(T)}
\end{aligned}
$$

which gives

$$
\frac{1}{G_{\tilde{\mu}}(t)}=\frac{1}{y} \exp \left(\int_{0}^{t} \frac{b(s)}{2 \sigma(s)} d B(s)+\frac{1}{2}\left(\frac{b(s)}{2 \sigma(s)}\right)^{2} d s\right)
$$

i.e.

$$
d\left(\frac{1}{G_{\tilde{\mu}}(t)}\right)=\frac{1}{G_{\tilde{\mu}}(t)}\left[\frac{b(t)}{2 \sigma(t)} d B(t)+\left(\frac{b(t)}{2 \sigma(t)}\right)^{2} d t\right] .
$$

We see that (3.47) is in agreement with (3.49) with $p_{2}(t)=\frac{1}{G_{\tilde{\mu}}(t)}$, and this proves that $\tilde{\mu}(t)$ given by (3.44) is indeed optimal also when $b$ and $\sigma$ are stochastic. The corresponding optimal portfolio for the robust utility maximization problem with initial value $x=\frac{1}{y}$, is, by (3.26),

$$
\hat{\varphi}(t)=\frac{b(t)}{G_{\tilde{\mu}}(t) 2 \sigma^{2}(t) S_{\tilde{\mu}}(t)} ; \quad t \in[0, T] .
$$

which means that the optimal fraction of wealth to be placed in the risky asset is

$$
\hat{\pi}(t)=\frac{\hat{\varphi}(t) S_{\tilde{\mu}}\left(t^{-}\right)}{\hat{X}(t)}=\frac{b(t)}{2 \sigma^{2}(t)}
$$

We have thus proved:

Proposition 3.6 Suppose (3.43) holds. Then the optimal scenario $\hat{\mu}=\tilde{\mu}$ and optimal portfolio $\hat{\varphi}$ for the robust primal problem (3.37) are given by (3.44) and (3.50), respectively, with $G_{\tilde{\mu}}(t)$ as in (3.45)).

Remark 3.7 Comparing (3.51) with the solution of the Merton problem in the classical, nonrobust case, we see that the optimal fraction to be placed in the risky asset in the robust case is just half of the optimal fraction in the non-robust case.

Example 3.2 We consider a robust version of Example 2.1. In this case the perturbed price $S=S_{\mu}$ is

$$
d S(t)=S\left(t^{-}\right)[(b(t)+\mu(t)) d t+\gamma(t, 1) d \tilde{N}(t)] ; S(0)>0
$$

and the wealth process $X=X_{\varphi, \mu}^{x}$ associated to a portfolio $\varphi$ is

$$
d X(t)=\varphi(t) S\left(t^{-}\right)[b(t) d t+\gamma(t, 1) d \tilde{N}(t)] ; X(0)=x>0 .
$$


We again choose the logarithmic utility function $U(x)=\ln (x)$ and the quadratic penalty function $\rho(x)=\frac{1}{2} x^{2}$.

Thus the robust primal problem is to find $(\hat{\varphi}, \hat{\mu}) \in \mathcal{A} \times \mathbb{M}$ such that

$$
\left.\left.\inf _{\mu \in \mathbb{M}} \sup _{\varphi \in \mathcal{A}} E\left[\ln X_{\varphi, \mu}^{x}(T)\right)+\int_{0}^{T} \mu^{2}(t) d t\right]=E\left[\ln X_{\hat{\varphi}, \hat{\mu}}^{x}(T)\right)+\int_{0}^{T} \hat{\mu}^{2}(t) d t\right] .
$$

The corresponding dual problem is to find $(\tilde{\theta}, \tilde{\mu}) \in \Lambda$ such that

$$
\sup _{(\theta, \mu) \in \Lambda} E\left[\ln \left(G_{\theta, \mu}^{y}(T)\right)-\int_{0}^{T} \mu^{2}(t) d t\right]=E\left[\ln \left(G_{\tilde{\theta}, \tilde{\mu}}^{y}(T)\right)-\int_{0}^{T} \tilde{\mu}^{2}(t) d t\right] .
$$

First note from (3.14) that for each $\mu$ there is only one admissible element process $\theta$ given by

$$
\theta_{1}(t, 1)=\tilde{\theta}_{1}(t, 1)=-\frac{b(t)+\mu(t)}{\lambda \gamma(t, 1)} .
$$

Assume that (see (2.9))

$$
\frac{b(t)+\mu(t)}{\lambda \gamma(t, 1)}<1 ; t \in[0, T]
$$

Then we get

$$
\begin{aligned}
G_{\tilde{\theta}_{1}, \tilde{\mu}}^{y}(t) & =y \exp \left(\int_{0}^{t} \ln \left(1-\frac{b(s)+\tilde{\mu}(s)}{\lambda \gamma(s, 1)}\right) d \tilde{N}(s)\right. \\
& \left.+\lambda \int_{0}^{t}\left\{\ln \left(1-\frac{b(s)+\tilde{\mu}(s)}{\lambda \gamma(s, 1)}\right)+\frac{b(s)+\tilde{\mu}(s)}{\lambda \gamma(s, 1)}\right\} d s\right) ; 0 \leq t \leq T .
\end{aligned}
$$

In this case, $K\left(q_{2}, r_{2}\right)(t)=\frac{r_{2}(t, 1)}{\gamma(t, 1)}$ and the BSDE (3.17) becomes:

$$
\left\{\begin{array}{l}
d p_{2}(t)=\frac{r_{2}(t, 1)}{\gamma(t, 1)}[b(t)+\tilde{\mu}(t)] d t+\int_{\mathbb{R}} r_{2}(t, 1) d \tilde{N}(t) ; t \in[0, T] \\
p_{2}(T)=\frac{1}{G_{\tilde{\theta}, \tilde{\mu}}^{y}(T)} .
\end{array}\right.
$$

To solve this equation, we proceed as in Example 2.1. We then get:

$$
\hat{p}_{2}=\frac{1}{G_{\tilde{\theta}, \tilde{\mu}}^{y}} ; \hat{r}_{2}=\hat{p}_{2} \psi
$$

with $\psi$ given by

$$
\psi(t)=\frac{b(t)+\tilde{\mu}(t)}{\lambda \gamma(t, 1)-(b(t)+\tilde{\mu}(t))}, t \in[0, T] .
$$

From (3.19), we get the equation:

$$
\tilde{\mu}(t)=-\frac{G_{\tilde{\theta}, \tilde{\mu}}^{y}(t) \hat{p}_{2}(t) \psi(t)}{\gamma(t, 1)}=-\frac{b(t)+\tilde{\mu}(t)}{\gamma(t, 1)(\lambda \gamma(t, 1)-(b(t)+\tilde{\mu}(t)))},
$$


i.e.

$$
\gamma(t, 1) \tilde{\mu}^{2}(t)+\left(\gamma(t, 1) b(t)-\lambda \gamma^{2}(t, 1)-1\right) \tilde{\mu}(t)-b(t)=0 .
$$

The root of this quadratic equation which satisfies (3.57) is

$$
\tilde{\mu}(t)=\frac{1}{2 \gamma(t, 1)}\left(-\gamma(t, 1) b(t)+\lambda \gamma^{2}(t, 1)+1-\sqrt{\Delta}\right) .
$$

with

$$
\begin{aligned}
\Delta & =\left(\gamma(t, 1) b(t)-\lambda \gamma^{2}(t, 1)-1\right)^{2}+4 \gamma(t, 1) b(t) \\
& =\left(\gamma(t, 1) b(t)-\lambda \gamma^{2}(t, 1)+1\right)^{2}+4 \lambda \gamma^{2}(t, 1) .
\end{aligned}
$$

From Theorem 3.5 we conclude that the solution of the robust primal problem (3.54) is $\hat{\mu}(t)=\tilde{\mu}(t)$ given by $(3.63)$, and

$$
\hat{\varphi}(t)=\frac{\hat{r}_{2}(t)}{\gamma(t, 1) S\left(t^{-}\right)}=\frac{\hat{p}_{2}(t) \psi(t)}{\gamma(t, 1) S\left(t^{-}\right)}=\frac{X_{\hat{\varphi}, \hat{\mu}}^{x}(t) \psi(t)}{\gamma(t, 1) S\left(t^{-}\right)}
$$

with $x=\frac{1}{y}$. The optimal fraction of the wealth invested in the risky asset is

$$
\hat{\pi}(t)=\frac{\hat{\varphi}(t) S\left(t^{-}\right)}{X_{\hat{\varphi}, \hat{\mu}}^{x}(t)}=\frac{\psi(t)}{\gamma(t, 1)}=\frac{b(t)+\hat{\mu}(t)}{\gamma(t, 1)(\lambda \gamma(t, 1)-(b(t)+\hat{\mu}(t)))}=-\hat{\mu}(t) .
$$

We summarize this as follows:

Proposition 3.8 The optimal pair $(\hat{\mu}, \hat{\varphi}) \in \mathcal{A} \times \mathbb{M}$ for Problem 3.54 is given by $\hat{\varphi}(t)=$ $\frac{\hat{\pi}(t) X_{\hat{\varphi}, \tilde{\mu}}^{x}(t)}{S\left(t^{-}\right)}$with $\hat{\pi}(t)=-\tilde{\mu}(t)$ and $\tilde{\mu}(t)$ given by $(\underline{3.63)})$.

\section{A Maximum principles for optimal control}

Consider the following controlled stochastic differential equation

$$
\begin{aligned}
d X(t) & =b(t, X(t), u(t), \omega) d t+\sigma(t, X(t), u(t), \omega) d B(t) \\
& +\int_{\mathbb{R}} \gamma(t, X(t), u(t), \omega, \zeta) \tilde{N}(d t, d \zeta) ; 0 \leq t \leq T ; X(0)=x \in \mathbb{R} .
\end{aligned}
$$

The performance functional is given by

$$
J(u)=E\left[\int_{0}^{T} f(t, X(t), u(t), \omega) d t+\phi(X(T), \omega)\right]
$$

where $T>0$ and $u$ is in a given family $\mathcal{A}$ of admissible $\mathcal{F}$-predictable controls. For $u \in \mathcal{A}$ we let $X^{u}(t)$ be the solution of (A.1). We assume this solution exists, is unique and satisfies

$$
E\left[\int_{0}^{T}\left|X^{u}(t)\right|^{2} d t\right]<\infty
$$


We want to find $u^{*} \in \mathcal{A}$ such that

$$
\sup _{u \in \mathcal{A}} J(u)=J\left(u^{*}\right) .
$$

We make the following assumptions

$$
\begin{array}{r}
f \in C^{1} \text { and } E\left[\int_{0}^{T}|\nabla f|^{2}(t) d t\right]<\infty, \\
b, \sigma, \gamma \in C^{1} \text { and } E\left[\int_{0}^{T}\left(|\nabla b|^{2}+|\nabla \sigma|^{2}+\|\nabla \gamma\|^{2}\right)(t) d t\right]<\infty, \\
\text { where }\|\nabla \gamma(t, \cdot)\|^{2}:=\int_{\mathbb{R}} \gamma^{2}(t, \zeta) \nu(d \zeta) \\
\phi \in C^{1} \text { and for all } u \in \mathcal{A}, E\left[\phi^{\prime}(X(T))^{2}\right]<\infty .
\end{array}
$$

Let $\mathbb{U}$ be a convex closed set containing all possible control values $u(t) ; t \in[0, T]$.

The Hamiltonian associated to the problem (A.4) is defined by

$$
\begin{gathered}
H:[0, T] \times \mathbb{R} \times \mathbb{U} \times \mathbb{R} \times \mathbb{R} \times \mathcal{R} \times \Omega \mapsto \mathbb{R} \\
H(t, x, u, p, q, r, \omega)=f(t, x, u, \omega)+b(t, x, u, \omega) p+\sigma(t, x, u, \omega) q+\int_{\mathbb{R}} \gamma(t, x, u, \zeta, \omega) r(t, \zeta) \nu(d \zeta) .
\end{gathered}
$$

For simplicity of notation the dependence on $\omega$ is suppressed in the following. We assume that $H$ is Fréchet differentiable in the variables $x, u$. We let $m$ denote the Lebesgue measure on $[0, T]$.

The associated BSDE for the adjoint processes $(p, q, r)$ is

$$
\left\{\begin{array}{l}
d p(t)=-\frac{\partial H}{\partial x}(t)+q(t) d B(t)+\int_{\mathbb{R}} r(t, \zeta) \tilde{N}(d t, d \zeta) ; 0 \leq t \leq T \\
p(T)=\phi^{\prime}(X(T)) .
\end{array}\right.
$$

Here and in the following we are using the abbreviated notation

$$
\frac{\partial H}{\partial x}(t)=\frac{\partial H}{\partial x}(t, X(t), u(t)) \text { etc }
$$

We first formulate a sufficient maximum principle.

Theorem A.1 (Sufficient maximum principle) Let $\hat{u} \in \mathcal{A}$ with corresponding solutions $\hat{X}, \hat{p}, \hat{q}, \hat{r}$ of equations (A.1)-(A.8). Assume the following:

- The function $x \mapsto \phi(x)$ is concave

- (The Arrow condition) The function

$$
\mathcal{H}(x):=\sup _{v \in \mathbb{U}} H(t, x, v, \hat{p}(t), \hat{q}(t), \hat{r}(t, \cdot))
$$

is concave for all $t \in[0, T]$. 


$$
\sup _{v \in \mathbb{U}} H(t, \hat{X}(t), v, \hat{p}(t), \hat{q}(t), \hat{r}(t, \cdot))=H(t, \hat{X}(t), \hat{u}(t), \hat{p}(t), \hat{q}(t), \hat{r}(t, \cdot)) ; t \in[0, T] .
$$

Then $\hat{u}$ is an optimal control for the problem (A.4).

Next, we state a necessary maximum principle. For this, we need the following assumptions:

- For all $t_{0} \in[0, T]$ and all bounded $\mathcal{F}_{t_{0}}$-measurable random variables $\alpha(\omega)$ the control

$$
\beta(t):=\chi_{\left[t_{0}, T\right]}(t) \alpha(\omega)
$$

belongs to $\mathcal{A}$.

- For all $u, \beta \in \mathcal{A}$ with $\beta$ bounded, there exists $\delta>0$ such that the control

$$
\tilde{u}(t):=u(t)+a \beta(t) ; t \in[0, T]
$$

belongs to $\mathcal{A}$ for all $a \in(-\delta, \delta)$.

- The derivative process

$$
x(t):=\left.\frac{d}{d a} X^{u+a \beta}(t)\right|_{a=0},
$$

exists and belongs to $L^{2}(d m \times d P)$, and

$$
\left\{\begin{aligned}
d x(t) & =\left\{\frac{\partial b}{\partial x}(t) x(t)+\frac{\partial b}{\partial u}(t) \beta(t)\right\} d t+\left\{\frac{\partial \sigma}{\partial x}(t) x(t)+\frac{\partial \sigma}{\partial u}(t) \beta(t)\right\} d B(t) \\
& +\int_{\mathbb{R}}\left\{\frac{\partial \gamma}{\partial x}(t, \zeta) x(t)+\frac{\partial \gamma}{\partial u}(t, \zeta) \beta(t)\right\} \tilde{N}(d t, d \zeta) \\
x(0) & =0
\end{aligned}\right.
$$

Theorem A.2 (Necessary maximum principle) The following are equivalent

$$
\begin{aligned}
& \text { - }\left.\frac{d}{d a} J(u+a \beta)\right|_{a=0}=0 \text { for all bounded } \beta \in \mathcal{A} \\
& \text { - } \frac{\partial H}{\partial u}(t)=0 \text { for all } t \in[0, T] .
\end{aligned}
$$

For proofs of these results we refer to Theorem 2.2 of [15]. 


\section{References}

[1] Bordigoni, G., Matoussi, A., Schweizer, M.: A stochastic control approach to a robust utility maximization problem. In: Benth, F.E. et al (eds):Stochastic Analysis and Applications. The Abel Symposium 2005, pp. 125-15, Springer (2007)

[2] El Karoui, N. and Quenez, M.-C.: Dynamic programming and pricing of contingent claims in an incomplete market. SIAM J. Control and Optimization 33 (1995), 29-66.

[3] El Karoui, N., Peng, S. and Quenez, M.-C.: Backward stochastic differential equations in finance. Mathematical Finance 7 (1997), 1-71.

[4] Föllmer, H., Schied, A., Weber, S.: Robust preferences and robust portfolio choice, In: Mathematical Modelling and Numerical Methods in Finance. In: Ciarlet, P., Bensoussan, A., Zhang, Q. (eds): Handbook of Numerical Analysis 15, pp. 29-88 (2009)

[5] Fontana, C., Øksendal, B., Sulem, A.: Viability and martingale measures in jump diffusion markets under partial information. Methodology and Computing in Applied Probability. DOI 10.1007/s11009-014-9397-4 (January 2014)

[6] Gushkin, A. : Dual characterization of the value function in the robust utility maximization problem. Theory Probab. Appl. 55 (2011), 611-630.

[7] Jeanblanc, M., Matoussi, A., Ngoupeyou, A.: Robust utility maximization in a discontinuous filtration, arXiv (2012)

[8] Kramkov, D. and Schachermayer, W.: Necessary and sufficient conditions in the problem of optimal investment in incomplete markets. Ann. Appl. Probab. 13 (2003), 1504-1516.

[9] Kreps, D.: Arbitrage and equilibrium in economics with infinitely many commodities. J. Math. Economics 8, 15-35 (1981)

[10] Lim, T.,Quenez, M.-C.: Exponential utility maximization and indifference price in an incomplete market with defaults. Electronic J. Probability 16, 1434-1464 (2011)

[11] Loewenstein, M., Willard, G.: Local martingales, arbitrage, and viability. Economic Theory 16, 135-161 (2000)

[12] Maenhout, P.: Robust portfolio rules and asset pricing. Review of Financial Studies 17, 951-983 (2004)

[13] Øksendal, B., Sulem, A.: Applied Stochastic Control of Jump Diffusions. Second Edition, Springer (2007)

[14] Øksendal, B., Sulem, A.: Forward-backward stochastic differential games and stochastic control under model uncertainty. J. Optim. Theory Appl., DOI 10.1007/S10957-012-0166-7 (2012). 
[15] Øksendal, B., Sulem, A.: Risk minimization in financial markets modeled by Itô-Lévy processes.Africa Mathematika, 2014, DOI:10.1007/s13370-014-0248-9 .

[16] Quenez, M.-C.: Optimal portfolio in a multiple-priors model. In R.C. Dalang, M. Dozzi and F. Russo (editors): Seminar on Stochastic Analysis, Random Fields and Applications IV, Birkauser 2004, pp. 291-321.

[17] Quenez, M.-C., Sulem, A.: BSDEs with jumps, optimization and applications to dynamic risk measures. Stochastic Processes and their Applications, 123 (2013), 3328-3357.

[18] Royer, M.: Backward stochastic differential equations with jumps and related non-linear expectations. Stochastic Processes and Their Applications 116, 1358-1376 (2006)

[19] Rockafellar, R.T.: Convex Analysis. Princeton University Press (1970)

[20] S.H.Tang and X. Li: Necessary conditions for optimal control of stochastic systems with random jumps, SIAM J. Cont. and Optim. 32, (1994), 1447-1475. 